\title{
Treatment of a giant arteriovenous malformation associated with intracranial aneurysm rupture during pregnancy: A case report
}

\author{
JUNHUI CHEN, YUHAI WANG, PEIPEI LI, WEILIANG CHEN, \\ JINGXU ZHOU, XU HU, JIE ZHU and BINGJIE JIANG
}

\author{
Department of Neurosurgery, 101st Hospital of Chinese People's Liberation Army, Wuxi, Jiangsu 214044, P.R. China
}

Received May 25, 2015; Accepted June 6, 2016

DOI: $10.3892 /$ etm.2016.3505

\begin{abstract}
Arteriovenous malformations (AVMs) associated with aneurysm have rarely been reported in the literature. The present study reports the case of a 21-year-old pregnant female patient who presented with a subarachnoid hemorrhage and an intracranial hematoma located in the anterior end of the corpus callosum. Furthermore, an anterior cerebral aneurysm and an AVM were identified by digital subtraction angiography and magnetic resonance angiography. The aneurysm was clipped and the AVM was successfully removed by microsurgery. The diagnosis of AVM associated with an aneurysm was confirmed via intraoperative and postoperative pathological examinations. By performing a review of the current literature, issues and surgical considerations associated with AVM associated with aneurysm were analyzed.
\end{abstract}

\section{Introduction}

Arteriovenous malformations (AVMs) are uncommon vascular lesions that result from multiple abnormal connections between arteries and veins (1). The coexistence of AVMs and aneurysms has rarely been reported since its initial description in $1942(2,3)$. A prospective study of 678 patients reported that the rate of AVMs associated with aneurysms was $18 \%$ (4). Stapf et al (5) demonstrated that $25 \%$ of 463 patients with AVMs had coexisting aneurysms. Despite the disease having severe effects on the health of sufferers, the causes and underlying mechanisms remain unknown. There exists three hypotheses that explain the pathogenesis of the disease; however, the consensus is that hemodynamic mechanisms serve an important role (6). In addition, it has been suggested that the coexistence of these two types of vascular disease in one patient may be a coincidence or the result of a congenital vascular malformation (7).

Correspondence to: Dr Yuhai Wang, Department of Neurosurgery, 101st Hospital of Chinese People's Liberation Army, 101 North Xingyuan Road, Wuxi, Jiangsu 214044, P.R. China

E-mail: wangyuhai1516@163.com

Key words: arteriovenous malformation, aneurysm, treatment
Patients with an AVM and an aneurysm have been shown to have a greater risk of experiencing an intracerebral hemorrhage, as compared with patients with an AVM or an aneurysm alone $(8,4)$. Thompson et al $(6)$ reported that the rate of intracerebral hemorrhage in patients with AVMs and aneurysms was $27-62 \%$. Furthermore, Brown et al (9) demonstrated that the incidence of intracerebral hemorrhage was $7 \%$ per year in these patients, which was $1.7 \%$ for patients with AVM alone.

This dual vascular disease presents a management challenge. The present study reports the case of a young pregnant patient with combined AVM and aneurysms who presented with a subarachnoid hemorrhage (SAH) at the 101st Hospital of Chinese People's Liberation Army (Wuxi, China). The authors of the present study experienced severe challenges when attempting to deal with the giant AVM and aneurysm in terms of the technology and management.

\section{Case report}

A 21-year-old female was admitted to Lujiang People's Hospital (Lujiang, China) on 6th June 2014 due to a short-term history of a severe headache, nausea and vomiting, in the absence of an obvious cause. The patient had no previous history of trauma. A computed tomography (CT) head scan revealed an intracranial hematoma in the right frontal lobe, and a SAH (Fig. 1). The patient was transferred to the regional center hospital where a magnetic resonance angiography (MRA) detected an AVM with a diameter of $5 \mathrm{~cm}$ in the right corpus callosum. The nidus was fed by the branches of the right anterior cerebral artery (ACA) and the right middle cerebral artery (MCA). In addition, there were multiple large cortical venous drains, one of which drained blood to the superior sagittal sinus, and one which drained blood to the sagittal sinus. Furthermore, a 3.0-mm enlarged blood vessel corresponding to an aneurysm was observed, although its existence could not be confirmed by an MRA (Fig. 2).

Digital subtraction angiography (DSA) confirmed the existence of a large AVM located in the right corpus callosum with multiple feeders from all branches of the ACA and MCA, and with drainage into the cavernous sinus and transverse sinus via deep temporal cortical veins. Furthermore, DSA clearly demonstrated an ACA aneurysm with a diameter of $3.0 \mathrm{~mm}$ adjoining the AVM (Fig. 3). Since the aneurysm was adjoining the AVM, it was difficult to evaluate the reason for the hemorrhage using CT scans. Therefore, the AVM and aneurysm 


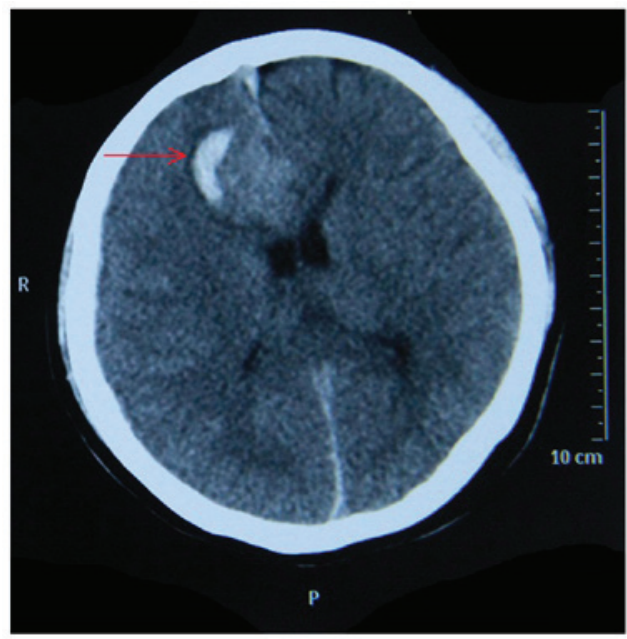

Figure 1. Preoperative computed tomography scan showed an intracranial hematoma in the right frontal lobe (red arrow) and a subarachnoid hemorrhage.

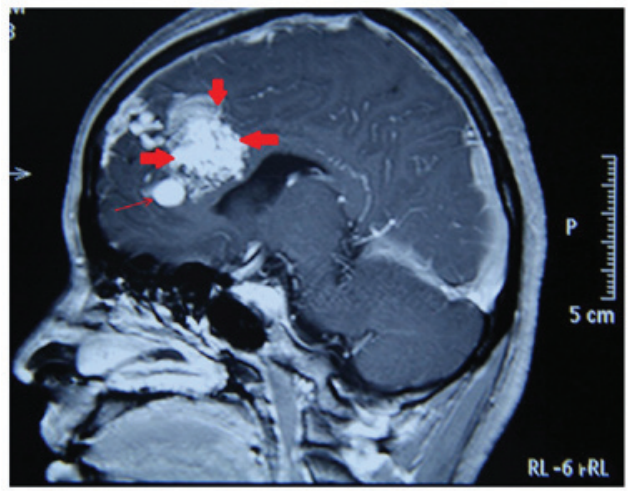

Figure 2. Preoperative magnetic resonance imaging demonstrated an arteriovenous malformation (thick red arrows) and associated aneurysm (thin red arrow).

were treated simultaneously to avoid re-bleeding. The hospital determined that the operation was too risky and the patient was admitted to the 101st Hospital of Chinese People's Liberation Army on day 15 following initial admission. A neurological examination involving use of the Glasgow Coma Scale (GCS) (10) detected no abnormalities (GCS score $=15$ ), and a CT reexamination showed that the $\mathrm{SAH}$ and intracranial hemorrhage blood had been absorbed.

A discussion involving neurology, neurosurgery and interventional radiology doctors determined that interventional vascular treatment was too risky, whereas microsurgery was considered a relatively safe method for treatment of the patient. The following day, the patient underwent a right pterional craniotomy, during which the bilateral A2 segment of the ACA was investigated using a longitudinal fissure approach to temporarily occlude the blood flow, while protecting the venous drainage system. The aneurysm was clipped completely after the parent arteries, aneurysm and main feeders of the AVM had been thoroughly explored. Subsequently, the right A3 segment was temporarily occluded (for $15 \mathrm{~min}$, followed by release for $10 \mathrm{~min}$ ) to explore the AVM, after which the part of the left A3 branches feeding the AVM were also clipped. Finally, the AVM was clipped integrally.
A

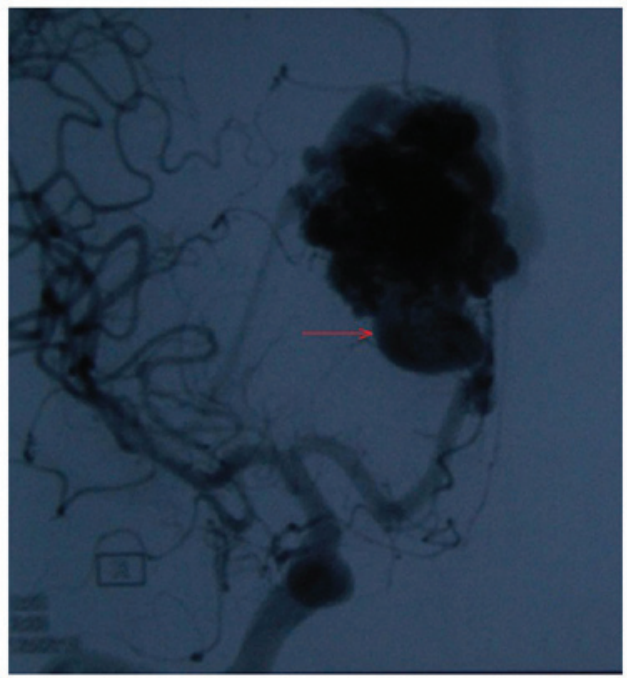

B

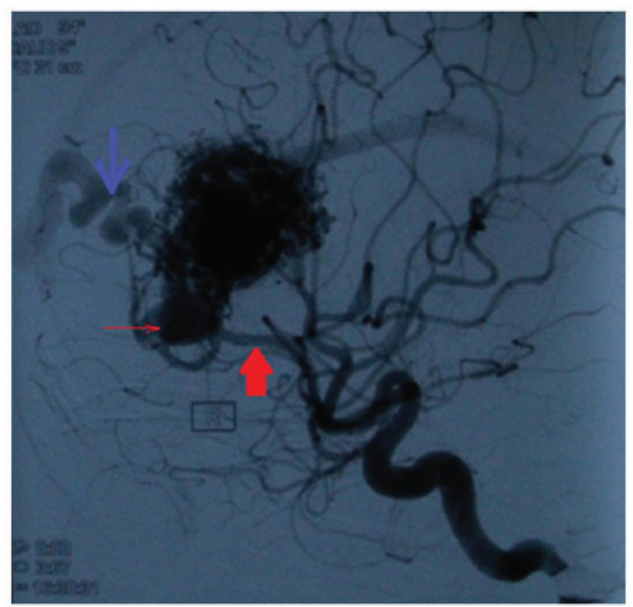

Figure 3. Preoperative digital subtraction angiography confirmed the results of the computed tomography scan and magnetic resonance imaging. (A) Venous phase, a large AVM and aneurysm are present, and the thin red arrow points to the aneurysm; (B) arterial phase, the blue arrow points to the draining vein, the thick red arrow points to the feeding artery and the thin red arrow points to the aneurysm.

A pathological analysis (Fig. 4), performed as previously described (11), showed that the AVM was $5 \times 4 \times 4 \mathrm{~cm}$ (Spetzler-Martin grade 4) (12). Postoperative control of the patient's blood pressure was important and it was maintained below the normal $\left(10-20 \mathrm{mmH}_{2} \mathrm{~g}\right.$ ) (normal systolic pressure, 120-140 mmHg; normal diastolic pressure, $70-90 \mathrm{mmHg}$ ). The patient was discharged from the Neonatal Intensive Care Unit on 20th June 2014 after 3 days with a GCS score of 15/15. No nerve dysfunction was observed. A CT and CT angiography (Fig. 5) was performed and demonstrated that the surgery had been successful, that the aneurysm had been clipped completely, that the AVM had been entirely removed and that no venous drain had been injured. At the 6 month follow-up, the Glasgow Outcome Scale (13) score was 5. Informed consent was obtained from the patient.

\section{Discussion}

The coexistence of AVMs and cerebral aneurysms has rarely been reported. The annual incidence of spontaneous SAH is $10 / 100,000$ worldwide, of which $75 \%$ are caused by intracranial 


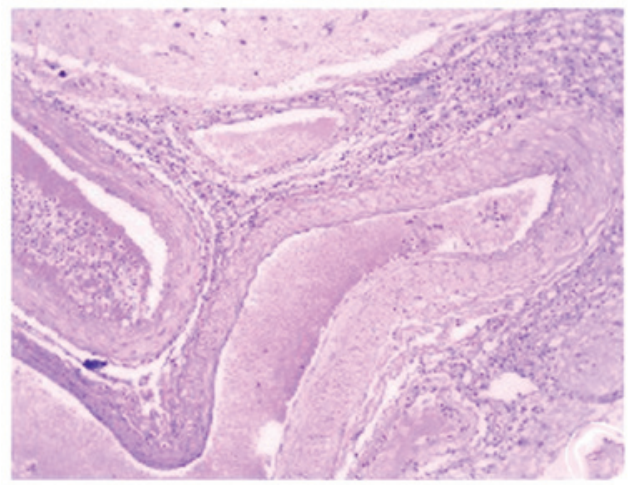

Figure 4. Postoperative histopathological examination showed abnormal, tortuously distributed vessels characteristic of an arteriovenous malformation.

A

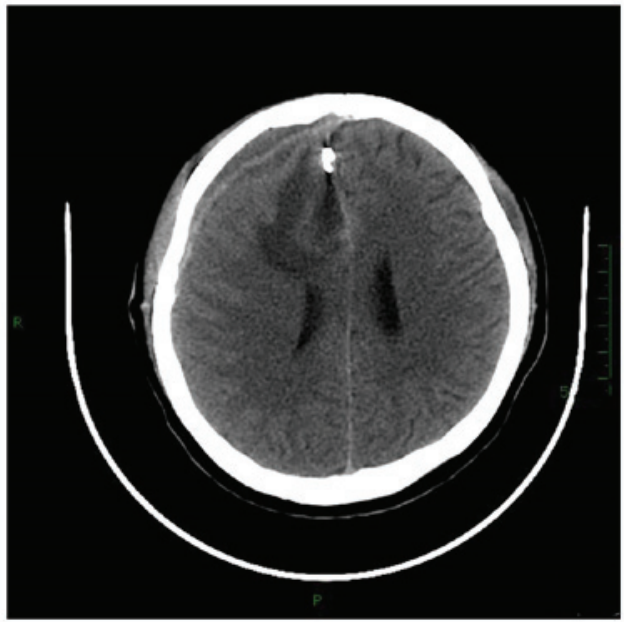

B

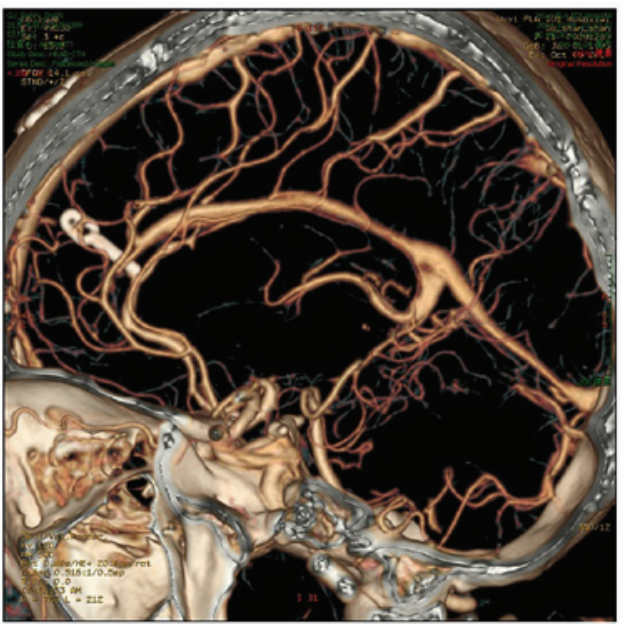

Figure 5. (A) Postoperative computed tomography (CT) scan showed that the hematoma and arteriovenous malformation had been completely removed (B) Postoperative CT angiography demonstrated that the aneurysm had been clipped.

aneurysms (14,15). The incidence of AVM is 1.3/100,000 per year (16), and the reported incidence of AVM coexisting with intracranial aneurysm is $18 \%$ (4). Therefore, the incidence of aneurysms in patients with AVMs is higher, as compared with general patients. Furthermore, the risk of bleeding and re-bleeding are higher in patients with AVM and aneurysm, as compared with patients with either alone $(8,4)$. In addition, in these patients, the risk of experiencing a hemorrhage increases over time (4). Marks et al (17) reported that aneurysms are an independent risk factor for re-bleeding. Therefore, it is important that microsurgery or endovascular treatment are performed early.

There are a number of systems used for the classification of AVMs associated with aneurysms; however, all systems are based on the location of the AVM relative to the aneurysm. The most commonly used classification system at present is that proposed by Perata et al (18). The patient in the present study was diagnosed with a flow-related aneurysm (type 2 classification). The position of the AVM relative to the aneurysm is important for deciding the appropriate treatment strategy and surgical approach. There has been significant debate regarding the most appropriate therapeutic strategy for AVMs associated with an aneurysm (19-23). Cunha et al (20) advised that priority treatment should be given to the symptomatic lesion or that both lesions should be treated simultaneously if the condition permits. Batjer et al (22) suggested that an intracranial aneurysm should be removed by microsurgery or endovascular embolization prior to resection of the AVM, since it may avoid rupturing the aneurysm during the surgery to remove the AVM. Conversely, Koulouris and Rizzoli (23) insisted that the AVM be removed first. Since numerous scholars consider that hemodynamic mechanisms serve an important role in the pathogenesis of the disease, the removal of AVM may lead to various hemodynamic changes causing the aneurysm to disappear spontaneously $(6,7,9,14,20)$. In the present case, the aneurysm and AVM were adjacent and the feeding artery of the AVM was the parent artery of the aneurysm. Therefore, the AVM and aneurysm could be removed simultaneously in the same operation, according to Cunha et al (20). However, the aneurysm was clipped prior to removing the AVM in order to reduce the risk of the aneurysm rupturing during surgery.

At present, three treatment strategies have been described for patients with coexisting AVM and aneurysm, including microsurgery, endovascular embolization and radiotherapy. Factors, such as the size and location of the AVM, venous drainage, hospital equipment and the technique level of the surgeon, will determine the requirement for treatment and the optimum therapeutic strategy. In the present study, microsurgery was used to remove the AVM and clip the aneurysm entirely. As a result, the prognosis of the patient was good and the intracranial hematoma was cleared. However, if the lesions had been deeply seated or located within an important functional area, microsurgery treatment may not have been the ideal choice (21).

Previous studies have reported that embolization has a number of advantages, including minor damage to brain tissue, fewer symptoms, effectiveness, rapid recovery and a short hospital stay (24-26). A previous study used gamma knife radiotherapy for treatment (27); however, it has a long treatment cycle, its effectiveness has not been well documented and re-bleeding may occur $(28,29)$. The present study describes a young pregnant woman with a large AVM, wide draining veins and a high risk of endovascular complications. Therefore, microsurgery was selected for treatment of the patient. A CTA reexamination demonstrated that the operation was 
successful; the AVM had been removed completely and the aneurysm was clipped.

Previous studies have not reported particular technical difficulties associated with the removal or embolization of giant AVMs coexisting with an aneurysm. However, it is important to protect the draining veins and arteries supplying blood to the brain tissue via the AVM. Furthermore, it is important that the intraoperative and postoperative blood pressure of the patient is maintained relatively low in order to reduce the risk of re-bleeding.

In conclusion, AVMs associated with intracranial aneurysms are a complex lesion, and the treatment is dependent on the relative location of each lesion and its classification. In the present study, a patient with a type 2 AVM and associated aneurysm underwent aneurysm clipping, followed by resection of the AVM in a single operation, and showed a good clinical outcome.

\section{References}

1. Richter GT and Friedman AB: Hemangiomas and vascular malformations: current theory and management. Int J Pediatr 2012: 645678, 2012.

2. Halim AX, Singh V, Johnston SC, Higashida RT, Dowd CF, Halbach VV, Lawton MT, Gress DR, McCulloch CE and Young WL; UCSF BAVM Study Project. Brain Arteriovenous Malformation: Characteristics of brain arteriovenous malformations with coexisting aneurysms: A comparison of two referral centers. Stroke 33: 675-679, 2002.

3. Walsh FB and King AB: Ocular signs of intracranial saccular aneurysms: Experimental work on collateral circulation through the ophthalmic artery. Arch Ophthalmol 27: 1-33, 1942.

4. da Costa L, Wallace MC, Ter Brugge KG, O'Kelly C, Willinsky RA and Tymianski M: The natural history and predictive features of hemorrhage from brain arteriovenous malformations. Stroke 40: 100-105, 2009.

5. Stapf C, Mohr JP, Pile-Spellman J, Sciacca RR, Hartmann A, Schumacher HC and Mast $\mathrm{H}$ : Concurrent arterial aneurysms in brain arteriovenous malformations with haemorrhagic presentation. J Neurol Neurosurg Psychiatry 73: 294-298, 2002.

6. Thompson RC, Steinburg GK, Levy RP and Marks MP: The management of patients with arteriovenous malformations and associated intracranial aneurysms. Ncurosurgery 43: 202-211; discussion 211-212, 1998.

7. Shen CC and Wang YC: Surgical management of intraeranial arteriovenous malformation associated with aneurysms. Zhonghua Yi Xue Za Zhi (Taipei) 61: 8-16, 1998 (In Chinese).

8. Wilkins RH: Natural history of intracranial vascular malformations: A review. Neurosurgery 16: 421-430, 1985.

9. Brown RD Jr, Wiebers DO and Forbes GS: Unruptured intracranial aneurysms and arteriovenous malformations: Frequency of intracranial hemorrhage and relationship of lesions. J Neurosurgery 73: 859-863, 1990.

10. Jennett B, Teasdale G, Braakman R, Minderhoud J and Knill-Jones R: Predicting outcome in individual patients after severe head injury. Lancet 1:1031-1034, 1976.

11. Martin NA and Vinters HV: Arteriovenous malformations. In: Neurovascular Surgery. Carter LP, Spetzler RF and Hamilton MG (eds). McGraw-Hill, New York, NY, pp875-903, 1995.

12. Spetzler RF and Martin NA: A proposed grading system for arteriovenous malformations. J Neurosurg 65:476-483, 1986.
13. Changaris DG, McGraw CP, Richardson JD, Garretson HD, Arpin EJ and Shields CB: Correlation of cerebral perfusion pressure and Glasgow Coma Scale to outcome. J Trauma 27: 1007-1013, 1987.

14. Steiner T, Juvela S, Unterberg A, Jung C, Forsting M and Rinkel G; European Stroke Organization: European stroke organization guidelines for the management of intracranial aneurysms and subarachnoid haemorrhage. Cerebrovasc Dis 35: 93-112, 2013.

15. Komotar RJ, Schmidt JM, Starke RM, Claassen J, Wartenberg KE, Lee K, Badjatia N, Connolly ES Jr and Mayer SA: Resuscitation and critical care of poor-grade subarachnoid hemorrhage. Neurosurgery 64: 397-410; discussion 410-411, 2009.

16. Gabriel RA, Kim H, Sidney S, McCulloch CE, Singh V, Johnston SC, Ko NU, Achrol AS, Zaroff JG and Young WL: Ten-year detection rate of brain arteriovenous malformations in a large, multiethnic, defined population. Stroke 41: 21-26, 2010.

17. Marks MP, Lane B, Steinberg GK and Chang PJ: Haemorrhage in intracerebral arteriovenous malformations: Angiographic determinants. Radiology 176: 807-813, 1990.

18. Perata HJ, Tomsick TA and Tew JM Jr: Feeding artery pedicle aneurysms: Association with parenchymal hemorrhage and arteriovenous malformation in the brain. J Neurosurg 80: 631-634, 1994.

19. Hoh BL, Putman CM, Budzik RF and Ogilvy CS: Surgical and endovascular flow disconnection of intracranial pial single-channel arteriovenous fistulae. Neurosurgery 49: 1351-1363; discussion 1363-1364, 2001.

20. Cunha e Sa MJ, Stein BM, Solomon RA and McCormick PC: The treatment of associated intracranial aneurysm and arteriovenous malformations. J Neurosurg 77: 853-859, 1992.

21. Passacantilli E, Pichierri A, Guidetti G, Santoro A and Delfini R: Surgical treatment of pial cerebellar arteriovenous fistulas with aneurysm of the main feeding artery. Surg Neurol 65: 90-94, 2006.

22. Batjer H, Suss RA and Samson D: Intracranial arteriovenous malformations associated with aneurysms. Neurosurgery 18: 29-35, 1986.

23. Koulouris $\mathrm{S}$ and Rizzoli HV: Coexisting intracranial aneurysm and arteriovenous malformations: Case report. Neurosurgery 8: 219-222, 1981.

24. Ibrahim T and Brophy BP: Subarachnoid haemorrhage in a patient with a left temporal arteriovenous malformation and an associated anterior communicating artery aneurysm. J Clin Neurosci 18: 1414-1416, 2011.

25. Kouznetsov E, Weill A, Ghostine JS, Gentric JC, Raymond J and Roy D: Association between posterior fossa arteriovenous malformations and prenidal aneurysm rupture: Potential impact on management. Neurosurg Focus 37: E4, 2014.

26. Meisel HJ, Mansmann U, Alvarez H, Rodesch G, Brock M and Lasjaunias P: Cerebral arteriovenous malformations and associated aneurysms: Analysis of 305 cases from a series of 662 patients. Neurosurgery 46: 793-800; discussion 800-802, 2000.

27. Gao E, Young WL, Pile-Spellman J, Joshi S, Duong H, Stieg PE and Ma Q: Cerebral arteriovenous malformation feeding artery aneurysms: A theoretical model of intravascular pressure changes after treatment. Neurosurgery 41: 1345-1356; discussion 1356-1358, 1997.

28. Grady C, Tanweer O, Zagzag D, Jafar JJ, Huang PP and Kondziolka D: Delayed hemorrhage from the tissue of an occluded arteriovenous malformation after stereotactic radiosurgery: Report of 3 cases. J Neurosurg 10: 1-6, 2016.

29. Lv X and Li Y: The clinical characteristics and treatment of cerebral AVM in pregnancy. Neuroradiol J 28: 385-388, 2015. 